\title{
Overcoming the Rayleigh Criterion Limit with Optical Vortices
}

\author{
F. Tamburini, G. Anzolin, G. Umbriaco, A. Bianchini, and C. Barbieri \\ Department of Astronomy, University of Padova, vicolo dell' Osservatorio 2, Padova, Italy.
}

\begin{abstract}
We experimentally and numerically tested the separability of two independent equally-luminous monochromatic and white light sources at the diffraction limit, using Optical Vortices (OV), related to the Orbital Angular Momentum (OAM) of light. The diffraction pattern of one of the two sources crosses a phase modifying device (fork-hologram) on its center generating the Laguerre-Gaussian (L-G) transform of an Airy disk. The second source, crossing the fork-hologram in positions different from the optical center, acquires different OAM values and generates non-symmetric L-G patterns. We formulated a criterion, based on the asymmetric intensity distribution of the superposed LG patterns so created, to resolve the two sources at angular distances much below the Rayleigh criterion. Analogous experiments carried out in white light allow angular resolutions which are still one order of magnitude below the Rayleigh criterion. The use OVs might offer new applications for stellar separation in future space experiments.
\end{abstract}

PACS numbers: 42.25.-p, 42.40.Eq, 42.40.Jv, 42.87.Bg, 07.60.-j

Introduction. - Electromagnetic (EM) radiation carries momentum: the linear momentum is usually associated with radiation pressure, while the angular momentum is associated with the polarization of the optical beam 11. However, the transfer of angular momentum of light to material bodies [2, 3] together with the creation of OVs have been demonstrated in several basic experiments, also in white light [4]. OAM of light cannot be ascribed only to a multipole component of the wavefront along the propagation direction [5, 6], but is also a quantum property of single photons [7, 8, 9]. OAM has also attracted attention for its applications in quantum communication 10, 11, 12] and in astronomy 13, 14, 15, 16]. The use of OV has recently allowed the separation of two monochromatic sources with $95 \%$ intensity contrast 17 .

The generation of beams carrying OAM proceeds thanks to the insertion in the optical path of a Phase Modifying Device (PMD) which imprints a certain vorticity on the phase distribution of the incident beam 10]. Consider a monochromatic pencil of light, described by an EM wave propagating along the $z$ direction of a reference frame $S$ and with transversal amplitude distribution $u(\mathbf{r})$. The PMD is placed on the $(x, y)$ plane and its optical singularity is centered on the origin $O$. When the incident beam has its propagation axis centered on the active region of the PMD, its Airy diffraction pattern is transformed into a series of donought-shaped concentric rings which do not carry net transverse momentum relative to their symmetry axis. Light beams carrying OAM are described by $\mathrm{L}-\mathrm{G}$ modes for which the EM field amplitude is

$$
\begin{aligned}
& u_{l p}^{L-G}(r, \varphi, z)=\sqrt{\frac{2 p !}{\pi(p+l) !}} \frac{1}{w(z)}\left[\frac{r \sqrt{2}}{w(z)}\right]^{l} \\
& \times L_{p}^{l}\left[\frac{2 r^{2}}{w^{2}(z)}\right] \exp \left[\frac{-r^{2}}{w(z)^{2}}\right] \exp \left[\frac{-\mathrm{i} k r^{2}}{2 R(z)}\right] \\
& \times \exp \left[-\mathrm{i}(2 p+l+1) \arctan \left(\frac{z}{z_{R}}\right)\right] e^{-\mathrm{i} l \varphi}
\end{aligned}
$$

where $z_{R}$ is the Rayleigh range of the beam, $w(z)$ the beam waist radius (the spot size), $L_{p}^{l}(x)$ an associated
Laguerre polynomial and $R(z)$ the radius of curvature. The azimuthal and radial indices $l$ and $p$ provide information about the OAM of the beam and the number of radial nodes of the associated intensity profile, respectively. As a consequence of the phase factor $\exp (\mathrm{i} l \varphi)$, any cylindrically symmetric L-G mode carries an OAM per photon, relative to its symmetry axis, equal to $l \hbar$. The intensity distribution of an $\mathrm{L}-\mathrm{G}$ mode with $p=0$ is, in polar coordinates, $I(r, \phi)=\frac{2}{\pi w^{2} l !}\left(\frac{r \sqrt{2}}{w}\right)^{2 l} \exp \left(\frac{-2 r^{2}}{w^{2}}\right)$, while the regions having the same intensity describe a ring structure. The center of the OV is dark, with a radius that, depending on the OAM value, reaches a maximum at $r_{\max }=\frac{\sqrt{2}}{2} w \sqrt{l}$, while the intensity scales as $I\left(r_{\max }\right)=\frac{2}{\pi w^{2} l !} l e^{l}$, which, for large values of the azimuthal index decreases as the inverse of the square root of $l[5]$. Strongly focused, tilted and off-axis beams are not described by a single donought-shaped L-G mode, but show a more complicated asymmetric pattern made by a superposition of an infinite number of coaxial $L-G$ modes. The OAM index of such a weighted superposition usually possesses a non-integer value 12, 18, 19, 20, 21].

The diffraction images of two point-like sources (Airy disks) are resolved when the maximum of intensity of one source overlaps the first intensity minimum of the second equally bright source. The historical definition 22] refers to a symmetrical double-peaked profile with a central dip $5 \%$ lower than the intensity maxima. For a telescope having diameter $D$, at wavelength $\lambda$, the separation is achieved at $\theta_{R}=1.22 \lambda / D$ [23]. Techniques to overcome this limit have been developed in certain special cases 24]. In 25 the separation of integer-valued L-G modes is obtained by measuring the distance of the maxima of an Airy disk centered on a L-G donought mode at distances different than the Rayleigh criterion. In particular, only for $l=1$ is achieved a sub-Rayleigh separation corresponding to 0.64 times $\theta_{R}$ [26]. Larger integer values of the azimuthal index $l$ produced separation angles 
larger than $\theta_{R}$.

In this paper, we show that the Rayleigh limit may be better overcome by using for the two sources a combination of integer and non-integer values of $l$.

Sub-Rayleigh separability. - In this section we present the experimental and numerical results, achieved using OVs, about the sub-Rayleigh separation of two equally intense and uncorrelated monochromatic sources and also experimental results obtained in white light. The optical scheme of the experiment is shown in Fig. 1 Two $632.8 \mathrm{~nm} \mathrm{He}-\mathrm{Ne}$ lasers generate the two independent monochromatic sources, while white light sources were provided by a halogen lamp and two optical fibers. Airy disks were produced by equal pairs of pinholes with diameters of 35, 50, 400 and $500 \mu \mathrm{m}$ placed at the same distance from the fork-hologram $\mathrm{H}$. During the experiment one beam was always coinciding with the optical center of the hologram, thus generating an OV with integer topological charge $l=1$. The other beam spanned the hologram in different positions starting from the optical center. For non-central positions the second beam formed an OV carrying non-integer components of OAM. This phenomenon is typical of laterally displaced and angularly deflected beams, which Airy disk transforms are composed of an infinite set of azimuthal harmonics expressed either in the form of Bonnet-Gaussian beams or with well-defined orbital and azimuthal values of $\mathrm{L}-\mathrm{G}$ modes [21, 27]. However, the central dark regions are always superposed, because they have been generated by the same central optical singularity of H. The separation of the diffraction figures of the two sources was obtained by using a moving beam splitter (MBS). The two beams were kept parallel with a tolerance of $10^{-5}$ degrees $(0.17$ $\mu \mathrm{rad}$ ), with negligible effects on the OAM value due to beam tilting. In white light we corrected the chromatic dispersion due to the hologram by spatially filtering the first generated diffraction order with the slit S placed on the Fourier plane of the achromatic lens L1 (see inset in Fig. 11). The hologram H, 20 lines $/ \mathrm{mm}$, has an active area of $2.6 \times 2.6 \mathrm{~mm}^{2}$ with a $50 \mu \mathrm{m}$-sized optical singularity. It is blazed at the first diffraction order and its efficiency is about $80 \%$ at the laser's wavelength. An incoming Gaussian beam is projected by $\mathrm{H}$ in a superposition of $\mathrm{L}-\mathrm{G}$ modes where the dominant modes have $l=0$ and $l=1$ for every value of $p$ 12]. $\mathrm{H}$ was placed perpendicular to the optical axis at a distance $d=430 \mathrm{~mm}$ away from the two pinholes, giving a Fresnel number $F \simeq 0.15$, sufficient to satisfy the Fraunhofer diffraction prescriptions to obtain Airy diffraction patterns on the hologram 23]. This was verified by inserting in the optical path a moving mirror (MM) at 45 degrees and analyzing the spots with the CCD1 camera (see Fig. 2 bottom row). By measuring the ratio of the distances of the first two diffraction pattern maxima with respect to the center, we obtained a value of 1.59 , close to 1.64 as provided by the theory.
In [25, 26] was discussed the separability of two identical overlapping OVs with the same OAM value: the integer-valued L-G transforms of the two Airy profiles were numerically computed for the same integer $l$ value and, following the Rayleigh separability criterion, the OVs of the two beams were rigidly superposed so that the maximum of one coincided with the dark center of the other. In this case, a sub-Rayleigh separation is achieved only when $l=1$ at an angular distance $\theta_{l=1}=0.64 \theta_{R}$. For OAM values $l>1$ the size of the OV becomes larger, scaling with $\sqrt{l}$, and two identical donought modes are separated at angular distances larger than $\theta_{R}$. For example, for $l=2$, the angular separation would be $\theta_{l=2}=1.03 \theta_{R}$, already worse than the Rayleigh criterion (see lower inset of Fig. [5]).

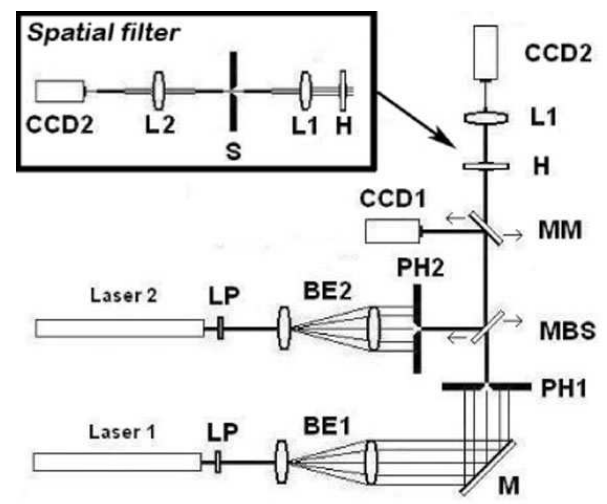

FIG. 1: Optical setup: LP's are neutral polarizing filters, BE1 and BE2 beam expanders, $\mathrm{M}$ a fixed mirror, MBS a moving beam splitter, MM a moving plane mirror, L1 a biconvex lens, $\mathrm{H}$ the fork-hologram, CCD1 and CCD2 two CCD cameras. The inset represents the spatial filtering for the white light: $\mathrm{L} 2$ is a camera lens and $\mathrm{S}$ a narrow slit (aperture $\lesssim 1 \mathrm{~mm}$ ). The two sources were obtained by using two distinct He-Ne lasers, in the monochromatic case, or a halogen lamp and two optical fibers in white light (not represented here).

The experimental results shown in Fig. 2 depict the sub-Rayleigh separability of two monochromatic OVs produced with our setup. Using the $400 \mu \mathrm{m}$ pinholes, the Rayleigh criterion limit was $\theta_{R}=1.93 \mathrm{mrad}$, corresponding to a linear separation $\delta_{R}=834 \mu \mathrm{m}$ on the hologram plane. The upper row of Fig. 2 2 shows the numerical simulations of $\mathrm{L}-\mathrm{G}$ modes generated by an $l=1$ forkhologram, the central row shows our experimental results and the bottom row shows the corresponding Airy figures of the two equally intense sources. The first column of Fig. 2 represents two coincident sources, the second column represents two sources separated by $0.42 \delta_{R}$ and the third column shows the sources separated by $0.84 \delta_{R}$.

In Fig. 3 we plot the intensity of the central section of the combined profiles of the L-G patterns along the direction connecting the two sources. The profiles are normalized with respect to the peaks of the superposed sources. We can observe that, as the linear separation 


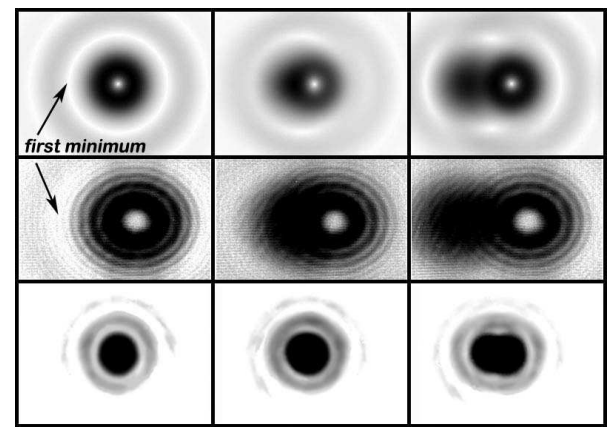

FIG. 2: Images of the separation of two nearby monochromatic sources having same intensity (inverted colors). Upper row: numerical simulations of $\mathrm{L}-\mathrm{G}$ modes generated by an $l=1$ fork-hologram at different separations. Central row: the corresponding experimental results. Bottom row: Airy patterns of the two sources on the hologram plane. Left column: the superposed sources. Mid column: the sources separated by 0.42 times the Rayleigh criterion. Right column: the sources separated by 0.84 times the Rayleigh criterion radius.

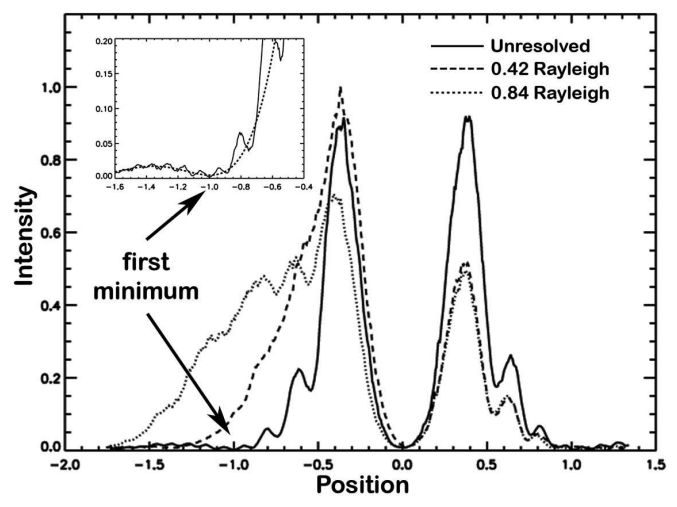

FIG. 3: Main figure: Experimental intensity profiles of the superposed $\mathrm{L}-\mathrm{G}$ modes of the two monochromatic sources normalized with respect to the peaks relative to coincident sources. The three cases shown refer to the same separations as in Fig. 2 When one of the two sources is shifted to an off-axis position the combined profile becomes clearly asymmetric. Inset: Zoom of the position of the first minimum of the $\mathrm{L}-\mathrm{G}$ transform of the Airy disk; the dotted curve represents the results of numerical simulations.

$\delta$ increases, the initially symmetric profile is distorted and the intensity of the two maxima assume different values. When $\delta=0$, the profile is symmetric and the two maxima have the same intensity given by the exact superposition of two identical $\mathrm{L}-\mathrm{G}$ modes. When $\delta=$ $0.42 \delta_{R}$, the intensity of one of the two maxima decreases much faster than the other one, the latter tending to reach the intensity level of the single source. In the limit $\delta \rightarrow \infty$ the on-axis beam still has $l=1$, but the off-axis one will assume $l \simeq 0$. For this reasons we devised a separability criterion based on the peaks' intensity ratio.

Fig. 4 plots the intensity taken at the first external

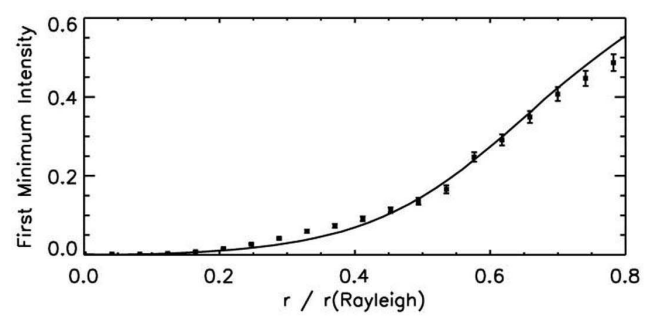

FIG. 4: The intensity of the first external minimum (see Fig. (3) of the combined profile produced by two equally intense sources vs the off-axis shift of one of them in units of the Rayleigh radius $\delta_{R}$. The continuous curve is derived from numerical simulations of the $\mathrm{L}-\mathrm{G}$ patterns produced by the two sources for different separations. Dots and error bars refer to our laboratory results. The intensity is normalized with respect to that of the two superposed sources.

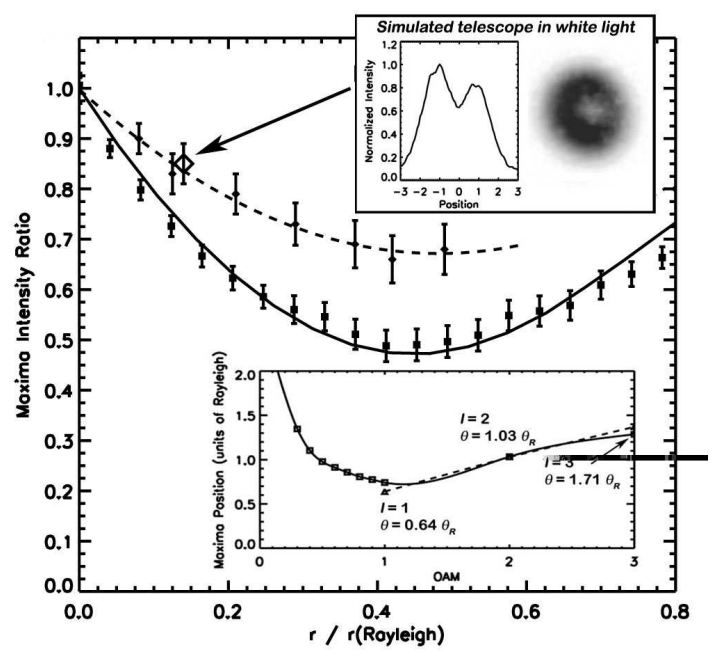

FIG. 5: Main figure: Ratio between the intensities of the peaks of the superposed $\mathrm{L}-\mathrm{G}$ modes vs the off-axis shift of the spot in units of the Rayleigh radius. The solid line represents the theoretical expectations for monochromatic light, while the superposed dots and errorbars are the experimental data. The dashed line is a linear interpolation of two experimental data obtained in white light. A $5 \%$ difference (see text) between the intensities of the peaks implies in both cases a separability at least one order of magnitude better than the Rayleigh limit. Lower inset: Positions of the maxima of the L- $\mathrm{G}$ modes relative to the OV (in units of the Rayleigh radius) vs OAM. Triangles show the angular separation values between two equally charged OVs as calculated in [25]. Upper inset: White light $\mathrm{L}-\mathrm{G}$ modes generated by two equally luminous simulated stars as seen with a diffraction limited telescope. The angular separation is $\sim 10$ times below the Rayleigh radius (empty diamond in the main figure).

minimum (as indicated in Fig. 31) of the combined profile produced by two equally intense sources vs the separation of the off-axis source. As the separation increases, the intensity taken at the position of the first external minimum monotonically increases due to the contribu- 
tion of the non-symmetric profile of the off-axis source with non-integer $\mathrm{L}-\mathrm{G}$ modes.

In Fig. 5 (main figure) we plot the intensity ratios of the main peaks produced by separated sources. We tested the separation of the off-axis monochromatic source in a range $0 \leq \delta \leq 700 \mu \mathrm{m}$, with a step of $35 \mu \mathrm{m}$. The experimental data show a good agreement with the theoretical curve obtained from numerical simulations of the $\mathrm{L}-\mathrm{G}$ transform of an Airy disk, following the mode decomposition shown in [12]. We see that the intensity ratios reach a minimum value 0.48 when the separation is $\sim 0.42 \delta_{R}$. By roughly assuming the Airy disk as a Gaussian one 28, we estimate the non-integer OAM value of the off-axis source at $\delta=0.42 \delta_{R}$ to be $l \simeq 0.4$. The lower inset of Fig. 5 reports the positions of the main peak of the simulated $\mathrm{L}-\mathrm{G}$ modes in units of the separation $\delta_{R}$ vs the estimated OAM values. We see that, considering only the positions of the intensity maxima, sub-Rayleigh separations are achieved only for $0.45<l<2$, with a minimum around $0.7 \delta_{R}$, which is still worse than the limit $0.64 \delta_{R}$ found in [25, 26]. In the same plot we also show the separations obtainable with the method discussed in 25] for two identically charged donoughts with $l=1,2,3$. Instead, if we analyze the relative intensities of the asymmetric peaks produced by the off-axis object, we may achieve a more efficient sub-Rayleigh separability limit. Obviously, the actual separability depends on the $\mathrm{S} / \mathrm{N}$ ratio of the data. The main figure also reports the data obtained in white light that suggest a different slope at small separations with respect to the monochromatic behavior. We might mimic the historical definition of the Rayleigh criterion by assuming that two identical sources are just resolved when the intensities of the asymmetric peaks differ by at least $5 \%$. In the monochromatic case, we would reach a theoretical separability 50 times better than the Rayleigh limit. Analogously, the results obtained in white light suggest a separability about 10 times better then the Rayleigh limit. This lower resolution is mainly due to the non-perfect spatial filtering and lower degree of coherence of the sources. In the upper inset of Fig. [5 we show a successful application of the separability criterion in white light, where we simulated the OVs of a double star with angular separation $\sim 10$ times below the Rayleigh limit, as seen with a diffraction limited telescope having the same focal ratio of the 122 cm Galileo telescope in Asiago.

Conclusions. - In this paper we have shown that when two sources cross an $l=1$ fork-hologram and one beam is always centered with the optical singularity, we obtain different $\mathrm{L}-\mathrm{G}$ patterns if the second beam is offcentered because it acquires a non-integer OAM value producing an asymmetric pattern. Using this property, we have shown both numerically and experimentally that both monochromatic and white light OVs can be used to reach separabilities at least one order of magnitude lower than the Rayleigh limit. Our results might have interesting applications in several techniques of applied optics and also in astronomy, especially in space missions and in telescopes with adaptive optics.

We would like to thank the Institut für Experimentalphysik, University of Wien, Zeilinger-Gruppe for support and helpful discussions and the three anonymous referees for their helpful comments. This work has been partly supported by ESO and by the University of Padova.

[1] L. Allen, M. W. Beijersbergen, R. J. C. Spreeuw, and J. P. Woerdman, Phys. Rev. A, 45, 8185, 1992.

[2] R. A. Beth, Phys. Rev., 50, 115, 1936.

[3] H. He, M. E. J. Friese, N. R. Heckenberg, and H. Rubinsztein-Dunlop, Phys. Rev. Lett., 75, 826, 1995.

[4] J. Leach, and M. J. Padgett, New J. Phys., 5, 154, 2003.

[5] M. J. Padgett, and L. Allen, Opt. Com., 121, 36, 1995.

[6] L. Allen, and M. J. Padgett, Opt. Com., 184, 67, 2000.

[7] J. Arlt, K. Dholakia, L. Allen, and M. J. Padgett, Phys. Rev. A, 59, 3950, 1999.

[8] G. Molina-Terriza, J. P. Torres, and L. Torner, Phys. Rev. Lett., 88, 013601, 2002.

[9] A. T. O'Neil, I. MacVicar, L. Allen, and M. J. Padgett, Phys. Rev. Lett., 88, 053601, 2002.

[10] J. Arlt, K. Dholakia, L. Allen, and M. J. Padgett, J. Mod. Opt., 45, 1231, 1998.

[11] A. Mair, A. Vaziri, G. Weihs, and A. Zeilinger, Nature, 412, 313, 2001.

[12] A. Vaziri, G. Weihs, and A. Zeilinger, J. Opt. B, 4, S47, 2002.

[13] M. Harwit, ApJ, 597, 1266, 2003.

[14] F. Tamburini, G. Umbriaco, G. Anzolin, C. Barbieri, and A. Bianchini, MSAIS, 9, 484, 2006.

[15] D. Mawet, P. Riaud, O. Absil, and J. Surdej, ApJ, 633, 1191, 2005.

[16] G. Foo, D. M. Palacios, G. A. Swartzlander Jr., Opt. Lett., 30, 3263, 2005.

[17] J. H. Lee, G. Foo, E. G. Johnson, and G. A. Swartzlander Jr., Phys. Rev. Lett., 97, 053901, 2006.

[18] G. Lenz, Opt. Com., 123, 423, 1996.

[19] L. E. Helseth, Opt. Com., 229, 85, 2004.

[20] J. E. Curtis, and D. G. Grier, Phys. Rev. Lett., 90, 133901, 2003.

[21] M. V. Vasnetsov, V. A. Pas'ko, and M. S. Soskin, New J. Phys., 7, 46, 2005.

[22] A. Danjon, and A. Couder, Lunettes et Teléscopes, Éditions de la Revue d'Optique Théorique et Instrumentale, Paris, 1935.

[23] E. Hecht, Optics 4th edition, Addison Wesley, San Francisco, 2002.

[24] H. Müller, S.-w. Chiow, Q. Long, C. Vo, and S. Chu, Opt. Lett., 30, 3323, 2005.

[25] G. A. Swartzlander Jr., Opt. Lett., 26, 497, 2001.

[26] D. Palacios, PhD Thesis, Worchester Polytechnic Institute, 2004.

[27] R. Zambrini, and S. M. Barnett, Phys. Rev. Lett., 96, 113901, 2006.

[28] S. R. Oemrawsingh, PhD Thesis, Leiden University, 2004. 